\title{
Contextos sociales y naturales de las Tradiciones peruanas
}

\author{
Lorenzo Huertas V. \\ Universidad Ricardo Palma, Lima - Perú \\ lhuertas@urp.edu.pe
}

\section{Resumen}

Este artículo resalta la adecuada y justa relación entre algunas tradiciones y los llamados lapsos críticos o tiempos de alteración genérica de la naturaleza; además, se enfatiza en los espacios y tiempos de las tradiciones basadas en hechos o eventos verdaderos sin alterar los contextos históricos y más bien enriqueciéndolos. En este caso, las tradiciones se convierten en una fuente histórica muy especial porque muestran sutilezas difíciles de alcanzar en otros testimonios históricos. El ordenamiento cronológico de eventos naturales catastróficos registrados en las tradiciones enriquece el cuadro general de los desastres en el Perú, desde el tiempo en que gobernaba el inca Pachacútec hasta tiempos en que vivió Palma. Gracias a las tradiciones, el historiador puede ver los hechos, acontecimientos y estructuras con sus rumores, colores, alegrías, llantos y quebrantos.

Palabras clave: Tradición, lapso crítico, contexto histórico, eventos naturales, catástrofe, Pachacútec.

\begin{abstract}
This article emphasizes the proper and fair relation between some Traditions and the so-called critical span or periods of natural generic alteration. Besides, it highlights the spaces and periods of Traditions based on facts or real events without altering the historical context, but rather enriching it. In this case, Traditions become a very special historical source because they display a finesse difficult to achieve in other historical testimonies. The chronological ordering of catastrophic natural events recorded in the traditions enhance the overall picture of Peruvian disasters, from when the Inca Pachacutec ruled to the times of Palma. Thanks to the Traditions, the historians can deal with both the facts, events and structures and with their rumours, colours, joys, tears and losses.
\end{abstract}

Keywords: Tradition, critical span, historical context, natural events, catastrophe, Pachacutec 
Lorenzo Huertas Vallejos, doctorado en la Universidad Mayor de San Marcos. Miembro de número de la Academia de Historia del Perú, profesor emérito de la Universidad Nacional San Cristóbal de Huamanga, miembro de número de la Sociedad Bolivariana del Perú. Miembro de número del Instituto Ricardo Palma. Director del Archivo de la URP. 


\section{Una invitación cordial}

Varias investigaciones sobre economía, religión y otros aspectos sociales de la historia del Perú registran la presencia recurrente de fenómenos naturales, sociales o antrópicos que alteraban, alteran y seguirán alterando el normal desarrollo del proceso histórico. Gracias a los aportes de los arqueólogos Luisa E. Wells (1990), Izumi Shimada (1991), Michael Moseley (1981) y otros, descubrimos y confirmamos que la presencia de estos fenómenos en los Andes es milenaria y que sucedían antes de que el hombre llegara a nuestro continente hace quince mil años. Además, nos enteramos de que su recurrencia y su no ciclicidad alteran la demografía, ocasionan retracción económica e influyen en la geopolítica, en los modelos habitacionales y en la ideología.

En la década del ochenta del siglo pasado, debido a la presencia del mega-Niño (1982-1983), los científicos comenzaron a estudiar la cronología de estos eventos y su tipología. En 1986 Anne Marie Hoquenghem, del Instituto Francés de Estudios Andinos, junto con Lub Orlieb establecieron una cronología y una tipología del fenómeno lluvioso. Un adelanto de este trabajo fue publicado ese mismo año por Hoquenghem en el libro titulado Para vencer a la muerte.

En investigaciones de ese tipo ocupábamos nuestro tiempo cuando el doctor Manuel Pantigoso, presidente del Instituto Ricardo Palma, nos extendió la cordial invitación a ser miembro de número de dicha institución académica; fue un alto honor aceptarla, pero sin duda había que involucrarse de manera directa en la temática palmista. Entonces nos asistió el recuerdo de que en algunas de sus tradiciones Ricardo Palma mencionaba erupciones volcánicas como las del Pichincha (Ecuador) en el siglo XVI, terremotos como el de Lima en 1746 y epidemias como la de 1856 que mató a 100.000 personas en el Cuzco. 
Dicho nombramiento nos obligó a revisar con más cuidado tanto las Tradiciones peruanas como el Epistolario. Con las referencias encontradas, ampliamos y enriquecimos nuestro conocimiento de aquellas coyunturas a veces catastróficas. Esta información, más la que durante años habíamos juntado, nos permitió observar con mayor claridad la cronología y tipología del fenómeno El Niño. La relectura de la obra de Palma nos planteó la siguiente pregunta: ¿qué pasaba en el resto del Perú cuando el norte del país se humedecía debido a la presencia del fenómeno lluvioso?

\section{Andando con las injurias}

Con la referencias de desastres naturales que Ricardo Palma anota en sus tradiciones, sumadas a otras provenientes de publicaciones especializadas y documentos directos, y tomando, además, como parámetro el fenómeno lluvioso conocido como El Niño, armamos la tabla general de alteraciones naturales y sociales en el Perú. En algunos casos, la presencia de estas alteraciones resultaba coetánea o cercana cronológicamente al fenómeno lluvioso, como sucedió en 1578 que hubo un mega-Niño y epidemias en el norte y al mismo tiempo sequía y epidemias en el Cuzco. Asimismo, hubo friajes en Apurímac y Arequipa, amén de alteraciones telúricas como terremotos, erupciones volcánicas, etc. Esto sucedía casi al mismo tiempo o dentro de un lapso de diez o quince años antes o después del fenómeno de El Niño. Bautizamos a este tiempo de confluencia de desastres como lapso crítico. La ocurrencia de estas alteraciones, como ya señalamos, anteceden a la presencia del hombre. Como historiador solo pudimos analizar estos eventos a partir siglo XIV d. C., tiempo en que gobernó Pachacútec. La indagación nos llevó hasta el siglo XX, secuencia dividida en cinco momentos de alteración general o lapsos críticos: 1) 1450-1490?, 2) 1575-1600, 3) 1719-1746, 4) 1856-1896, 5) 1970-1998. En cada uno de estos "momentos" encontramos una, dos o más tradiciones que mencionan la alteración. 
Para entender mejor las tradiciones que se enmarcan en tiempos de alteración natural o social, es necesario tener en cuenta los contextos históricos con que contó Palma para escribirlas. Por ejemplo, en la actualidad se conoce la gran mortandad que enlutó al espacio andino durante el gobierno de Pachacútec debido a una pandemia. El cronista Felipe Huamán Poma de Ayala comenta este lapso crítico en su Primer nueva crónica y buen gobierno del siguiente modo:

Había mucha mortanza de indios y hambre y sed y pestilencia y castigo de Dios, que no llovió -en el Cuzco- siete años, otros dicen que diez y habían tempestades lo más del tiempo era todo llorar y enterrar difuntos (1993, p. 86).

La historia memorial de los pueblos andinos como los de la provincia de Cajatambo achacaban esa desgracia a la profanación que sufrió la ciudad sagrada de Pachacamac cuando Pachacútec ordenó construir dentro de ella un tambo real; es decir, un núcleo urbano de carácter administrativo, religioso y militar; por eso, encontramos en dicha ciudad dos templos: uno dedicado a Pachacamac y otro dedicado al Sol o Intihuasi, "casa del Sol"; Además una zona residencial para vivienda de los mandos incas o incahuasi y un acllahuasi donde medio millar de mujeres se dedicaban a la producción de tejidos finos y a elaborar bebidas especiales como la coco asua, libada durante las ceremonias religiosas. También había espacios donde trabajaban artesanos dedicados al menaje de la clase dirigente y de las deidades. Un tambo real tenía también una zona de collcas, o silos, ubicadas en espacios especiales para el rápido servicios de las zonas de producción y reserva en tiempos de escasez; guardaban alimentos, vestuarios, zapatos y armas para los ejércitos.

La construcción de estas grandes edificaciones necesitó la concurrencia de miles de indios mitmas, la presencia masiva de gente alteró el normal ritmo de vida en Pachacamac. Este 
dinamismo humano compuesto por diferentes agrupaciones étnicas profanó los recintos sagrados de aquella ciudad y -según la ideología indígena- cuando esto sucedía, las consecuencias eran nefastas. El dios Pachacamac tenía encerradas a las enfermedades en recintos de aquella ciudad; pero profanados estos espacios se disipó el vínculo entre los hombres y la deidad, los poderes de Pachacamac se relajaron y, en estas circunstancias, se escaparon las enfermedades que estaban encerradas en sitios seguros de aquel lugar e infectaron hasta los últimos rincones del antiguo Perú. Fue tiempo de "mortanza", la población andina colapsó (Duviols, 2003, p. 173).

Ricardo Palma, en su tradición "Los polvos de la condesa", afirma que murieron 30.000 soldados con ardientilla o fiebre amarilla. El número de víctimas es considerado como una exageración o como una licencia de Palma; pero, como se afirma, en el tiempo que gobernó Pachacútec se produjo un lapso crítico: hubo humedad y calentamiento genéricos, el espacio se llenó de miasmas y la muerte masiva fue tanta que la gente comentaba que parecía que la generación de los indios llegaba a su fin (Palma, 2014, p. 315).

Cuando nuestra tradicionalista escribió "Los polvos de la condesa", se desconocía el tema del cambio climático, sin embargo, la tradición calza plenamente con el tiempo, espacio y ambiente de dicho fenómeno. Otra consideración a tenerse en cuenta es la época en que Palma ubica a la tradición en el tiempo de los incas, especialmente de los incas imperiales, que coincide con la época en que Pachacútec ordenó el cambio de escala, es decir, el empleo de gran número de hombres en la guerra y en el levantamiento de obras como Machu Picchu, Sacsayhuamán, Ollantaytambo, Pachacamac, etc., y también en la construcción de andenes o canales como la Achirana en Ica.

El imaginario popular y el ingenio de Palma aderezaron estos hechos y describieron la construcción de estas grandes obras como actos de estrategia geopolítica o como antojos benignos 
del inca, a los que, por supuesto, adicionan a jóvenes doncellas en nombre de quienes el inca, en muestra de su cariño, mandó hacer la acequia llamada la Achirana. Estas dos tradiciones "Los polvos de la condesa" y "La Achirana del inca" están enmarcadas en el primer lapso crítico (1450-1490). Gracias a estas tradiciones se descubre aspectos relevantes en el desarrollo cultural durante el gobierno de Pachacútec y encajan a plenitud con la pandemia del siglo XIV que despobló al naciente imperio de los incas y obligó al cambio de escala en construcción de megaobras como la Achirana, lo mismo que en la guerra.

En cuanto al segundo lapso crítico (1575-1600), en la tradición "Cristo de la Agonía", Palma registra las erupciones volcánicas del Pichincha de 1534, 1539y 1577. En tanto que en la tradición "Cien por uno" se refiere a los efectos climáticos ocasionados por la erupción del Huaynaputina a inicios de febrero de 1600. Estas dos tradiciones señalan el inicio y el final del segundo lapso crítico, pero hay otras que dan marco a ese tiempo de alteración genérica.

$\mathrm{Al}$ respecto, en la tradición "El peje chico", Palma nos habla del saqueo hecho por españoles en las antiguas tumbas de Chan Chan el año de 1575 y de la profanación de las tumbas y otros espacio sagrados. Este suceso representó una catástrofe social para los indígenas de Trujillo del siglo XVI descendientes del Tacaynamo, quien fue el fundador de Chan Chan. Según creencias populares, cuando se producían estas profanaciones se rompían las vinculaciones entre los espíritus de hombres sagrados, cuyos cuerpos yacían en esas tumbas, eso ponía fin a las contraprestaciones entre los vivos y los muertos, y con ello la desgracia general llegaba.

Estas creencias explican lo acaecido en 1578, cuando el "cielo se desató" y parecía que llegaba el fin del mundo. Un megaNiño no visto ni recordado por humano alguno mató a miles de 
indígenas sobre todo niños y ancianos: la gente creía que llegaba el fin del mundo. Y en medio de ese chaparrón de desgracias, con colectividades asustadizas, abandonaron sus viejos cultos y se protegieron bajo la cruz cristiana; otros mantuvieron en silencio sus cultos antiguos en la clandestinidad y creció el apego a sus dioses gentilares. Ese año hubo sequía en el Cuzco y friaje en Puno, Cuzco y Apurímac; terremotos en Lima 1576, 1578, también en 1581 y 1587; en 1582 lo hubo en Arequipa; en 1586 en Trujillo, Lima, Arequipa e Ica; en 1594 en Ica y en 1598 en Lambayeque. Esta sismicidad se cierra con el gran terremoto y la erupción del volcán Huaynaputina que arruinó centros poblados de Arequipa, Moquegua y Tacna, fue una ruina general en el sur (v. Huertas, 2009).

Ricardo Palma en su tradición "Ciento por uno", situada en 1616, se refiere a un español llamado a Alonso Escoto que vivía asediado por la mala suerte, pero una tarde en que se halló solo frente a la Virgen de Copacabana le pidió prestados sus valiosos pendientes y dos candelabros de plata y prometió devolvérselos con creces. Escoto se dirigió a Arequipa, donde vendió los pendientes y los candelabros. Después lo encontramos en una viña arequipeña cuyo dueño se lamentaba diciendo que, por el cambio del clima causado por el volcán, su vino se había torcido y no lograba venderlo ni como vinagre. Compró aquel vinagre a precio irrisorio y partió con su cargamento a Lima, donde comprobó que aquel vinagre se había convertido en un generoso vino de primera calidad. Además, las antiguas historias cuentan que en ese año escaseaba el vino en Lima por el naufragio de naves que traían dicha bebida desde España, así que las ganancias de Escoto se quintuplicaron. Escoto devolvió con creces los pendientes y los candelabros de la Virgen de Copacabana. Las variables que sirvieron a Palma para crear dicha tradición son ciertas, pues erupcionó el Huaynaputina, hasta entonces Arequipa era la región de mayor producción de vinos y por esos años había un gran culto a la Virgen de Copacabana. 
No hemos encontrado un lapso crítico en el siglo XVII, falta más investigación aunque en las Tradiciones peruanas encontramos muchas referencias sobre terremotos de las que enumeraremos algunas. Por ejemplo, en la tradición "El virrey de los milagros" se menciona el gran terremoto del mes de noviembre de 1604 que destruyó Arequipa, Moquegua y se sintió en Ica, Lima y Ayacucho. Este gran movimiento ocasionó la migración de muchos vecinos de Arequipa; algo parecido sucedió en Ica. En "Tamborcito del Pirata" se menciona el terremoto que en octubre de 1609 sacudió Lima. Hay que indicar que en ese año aún el culto al Señor de los Milagros no se había entronizado en Lima, y era Santa Catalina la virgen que apaciguaba "la ira del Señor" e intercedía en esos momentos de dolor. En el mes de diciembre del mismo año, otro terremoto sacudió a la Ciudad Blanca. En la tradición "El Encapuchado", Palma anota el fortísimo sismo de 1650 que destruyó la ciudad del Cuzco, fue en ese conmovido tiempo cuando se entronizó en esta ciudad el Señor de los Temblores. Pero también aumentaron las muchas o adoraciones a la mama pacha para que se calmara y no hubiera más pachacuyun o terremotos. En las tradiciones "El virrey hereje" y "Un campanero bellaco" se menciona el terremoto de 1655; en la tradición "El que más vale no vale tanto como Valle vale", Palma menciona el terremoto de 1664. Como se puede ver, el siglo XVII fue la centuria de los terremotos, fue el siglo de cambios climáticos que influyeron en la producción. Por ejemplo, en los siglos XVI y XVII, la costa peruana, desde Motupe (en Lambayeque) hasta Ica, fue zona triguera, pero después de los sismos de 1682 y 1687 la costa dejó de producir trigo como antes.

Para el tercer lapso crítico (1719-1746) tenemos la tradición "Muerta en vida", en la que Palma menciona el eclipse del 15 de agosto de 1719. Como se sabe, en la ideología andina, este fenómeno, lo mismo que el paso de los cometas, eran preludio de futuras calamidades. Y vaya coincidencia, ese mismo año una pandemia mató a más de 200.000 mil personas en el Altiplano sureño y en el Cuzco; además, en 1720 y 1728 dos mega-Niños 
destruyeron centros poblados del norte del país. En 1725 hubo terremotos en Huaraz y Arequipa, y se cierra este lapso con el terremoto de 1746 que destruyó Lima y otros pueblos de la costa central. Palma los menciona en las tradiciones "El conde de la topada", "Callao y Chalaco" y "Mogollón".

Conviene hacer un alto en esta relación de catástrofes naturales registradas por Palma en su Tradiciones peruanas. Hasta aquí las referencias de Palma nos ayudaron a completar la tabla de los lapsos críticos desde el siglo XIV, con Pachacútec como gobernante del incario, hasta el cuarto lapso crítico que se inició en 1856 y se prolonga hasta fines del siglo XIX, alteración genérica de la que Ricardo Palma vivió una experiencia directa. Además, a mediados de esa centuria se produjo un cambio climático general, la tierra entró en un periodo geológico conocido como Optimun climáticum y con ello experimentó un cambio general del clima que coincide con una fase de la historia social conocida como Revolución Industrial; el capitalismo industrial se universaliza por el uso de maquinarias movidas a vapor. Como consecuencia, en el Perú aparecen los tranvías, ferrocarriles y los vapores que Palma utilizó tanto en Lima como cuando viajó a Europa. Aparecen los tractores movidos a vapor. Desaparecen los grandes bosques de huarangos en la costa central y los bosques de algarrobos del norte. Declinación de la industria vitivinífera y hay un apogeo del cultivo del algodón y la caña de azúcar, lo mismo que la explotación de la lana y el petróleo. También fue un tiempo de reacomodos geopolíticos en todo el mundo. Crecen las ciudades y la población mundial. Pero no todo fue canto de sirenas porque, como hemos dicho, empieza a alterarse la ecología mundial, los ríos que pasaban cerca de las minas fueron envenenados, desaparece su biomasa y la naturaleza vuelve a convulsionarse.

Palma en la tradición "El judío errante en el Cuzco" dice que en 1856 el tifus mató a cien mil personas en el Cuzco. En esta misma tradición, Palma menciona a un personaje macilento en 
Zurite, a quien y el pueblo le echó la culpa de tanta desgracias y le dio muerte ${ }^{1}$. Aquí hay que añadir que era costumbre inveterada hacer sacrificios humanos cuando ocurrían grandes desastres. En tiempos de Palma subsistía la costumbre de los pagapus y sacrificios de llamas y cuyes.

También con respecto al cuarto lapso crítico, Ricardo Palma menciona en su tradición "La conga" tres eventos importantes: 1) La lucha del coronel Balta contra Mariano Ignacio Prado, 2) La presencia de la fiebre amarilla que mató más gente que la misma guerra y 3) La conga, canción que se cantó y bailó haciendo marco al conflicto armado. Cabe señalar que el cuarto lapso crítico comenzó con el tifus en el Cuzco en 1856 y continuó después con la fiebre amarilla en Chiclayo que Palma recoge en esta tradición. Además se produjo en 1868 el terremoto más violento sucedido en el sur, que destruyó Arica, Moquegua y Arequipa. Asimismo, hubo dos Niños en 1887 y 1991 . Esta alteración finaliza con una larga sequía que comenzó después de El Niño de 1891 y prosiguió hasta 1919, con dos interrupciones en 1804 y 1811.

En conclusión, como se ha visto en el desarrollo de este artículo, las tradiciones escritas por Ricardo Palma constituyen una fuente histórica muy especial sobre los eventos naturales catastróficos acaecidos desde los tiempos en que gobernó el inca Pachacútec hasta los que vivió el autor. El seguimiento de los registros de fenómenos catastróficos en la obra de Palma nos permite una visión más amplia y completa del cuadro general de los desastres en el Perú.

l El sacrificio del judío errante en Zurite parece ser pura fábula; sin embrago, en 1975 recogimos relatos de pobladores del departamento de Ayacucho en los que se contaba que para evitar que las jarjachas y los pistacos hicieran daño a la colectividad, sacrificaron un niño opa o tonto. Tratamos de averiguar un poco más sobre esa habladuría, pero la gente hizo mutis y la noticia se desvaneció en las alturas de Huanta. 


\section{Bibliografía}

Duviols, P. (2003). Proceso y visitas de idolatrás. Cajatambo, Siglo XVII. Lima: PUCP. Fondo Editorial.

Hocqueghem, A. M. (1996). Para vencer a la muerte. Lima: IFEA INCAP- CNRS.

Huamán Poma de Ayala, F. (1993). Primer Nueva Coronica y Buen Gobierno. Edición y prólogo de Franklin Pease. T. I. México: FCE.

Huertas Vallejos, L. (2009). Injurias del tiempo. Desastres naturales en la historia del Perú. Lima: Editorial Universitaria de la URP.

Moseley, M. y Felmann R. (1981). "Vivir en crisis, percepción humana del proceso y tiempo". En Revista del Museo Nacional, t. XLVI, Lima.

Palma, R. (2014). Tradiciones Peruanas. Primera y Segunda series. Tomo I, Vol. I. Lima: Editorial Universitaria de la Universidad Ricardo Palma.

. (2015). Tradiciones Peruanas. Quinta y Sexta series. Tomo I, Vol. III. Lima: Editorial Universitaria de la Universidad Ricardo Palma.

. (2016). Tradiciones Peruanas. Séptima y Octava series. Tomo I, Vol. IV. Lima: Editorial Universitaria de la Universidad Ricardo Palma.

Shimada, I. (1991). "Implicaciones naturales de una gran sequía en el siglo VI en los andes peruanos”. En Boletín de Lima XIII (77).

Recibido el de marzo del 2019

Aceptado el de marzo del 2019 